\section{(Clintral alentures}

\author{
ON THE
}

\section{BORDERLAND OF EPILEPSY : \\ VERTIGO.}

Delivered at the National Hospital fOR the Paralysed and Epileptic.

By SIR WILLIAM R. GOWERS, M.D., F.R.S., PHYSICIAN TO THE HOSPITAL; CONSULTING PHYSICIAN TO UNIVERSITY COLLEGE HOSPITAL.

\section{LECTURE II.}

Gen'rnemen,-In the last lecture we considered the features of aural vertigo which resemble the symptoms of epileptic seizures-suddenness, brevity, loss of consciousness, loss of sight, and also the remarkable sense of impulsion downwards by an external force, sometimes so intense as to amount to a feeling that a blow has been received. The latter, although suggestive of an epileptic sensation, is probably peculiar to vertigo.

Other cephalic sensations, various in character, may attend the onset of the attack. Sometimes they are allied to that which we have just considered. A lady of 27, with the remains of otitis media on the right side and some labyrinthine deafness on the left, had also constant tinnitus, continuous, at times louder, and then pulsating like an engine. Her attacks of giddiness began with a sudden sensation in one side of the head, which seemed as if it would throw her down. But the sensation varied, and sometimes was "beating" in character-half sound and half pressurewhich made her hold her head firmly with her hands. Occasionally she woke up in the night with a loud noise like machinery in the right ear, and this seemed to pass gradually to the middle of the head and then to the left side; it lasted long-sometimes even twenty-four hoursand while it continued she could hear nothing. Of special interest is the fact that the sense of pressure seemed also to cause impulsion. These cephalic sensations, at the onset of an attack of vertigo, are sometimes very misleading. They are so obtrusive as to absorb the attention of the patient. In one instance of this, the aural origin of the symptoms rested on the timnitus, since hearing was rood-at least for a lady of 60 . For many months she had been troubled by a loud noise, referred to both ears, compared to the sound of a steam-engine at work. During the last three months there had been vertigo, moderate, often lasting several hours at a time, and apt to be especially severe on waking in the morning. Sometimes she dared not raise her head from the pillow. In the night it was often induced by turning over to the right, and obliged her to turn back to the left. (Such an effect of posture is always significant.) But she had also peculiar attacks, which generally occurred soon after rising in the morning. A sudden sensation from the back of the neck seemed to dart over the head, and a feeling as if she were losing consciousness made her throw herself on the bed; in a second or two she was all right again. She never became unconscious. These attacks afterwards ceased, but the slighter continuous giddiness still occurred at times. In this case, again, we cannot doubt that the symptoms were of aural origin, and the sense of impending unconsciousness was probably closely allied to vertigo. Yet, alone, it would strongly suggest epilepsy.

Another example of a cephalic sensation was afforded by a lady of 65 , completely deaf on the right side and partially on the left. She had suffered much from tinnitus, and for six months from slight prolonged giddiness, with a sense that objects were moving round her. But she had also sudden attacks after lying down at night, very similar in character to those of the case last described, but opposite in their relation to posture. She had a feeling of a sudden "rush" to the head, and that she would become unconscious if she did not instantly sit up. On doing so, the feeling ceased at once. The contrasted similarity of these two cases is remarkable. Each illustrates the effect of posture, and each shows also the prominent part a cephalic sensation may take in an attack which is essentially. vertiginous.
AtTaCks DURING SleEp.

Another feature presented in common by epilepsy and aural vertigo is the occurrence of attacks during sleep. It may seem surprising that such disturbance should occur during the cerebral quietude that constitutes sleep, but certain considerations may help us to understand it. That which disturbs sleep is sudden vertigo. The suddenness of the onset, as I have said, we must refer to the centre for equilibration, not to the peripheral processes which derange it. These seem to bring the centre into a state of instability, in which its balance suddenly gives way. During the recumbent posture assumed in sleep, the pressure in the semicircular canals is different from that caused by the upright position of the head. In the cases last considered we have seen the effect of this difference. If the change in the sensory elements of the canals is such that they are specially influerced when the posture is horizontal, their stimulation may gradually bring the equilibration centre beyond its stable point. Thus, the constant influence may overturn the balance, as we may often see in the illustration provided for us in the flushing cisterns, where a slow flow of water gradually raises a ball to a point at which it turns a stop, and the contents of the cistern suddenly escape. So far we may reasonably speculate. But there may well be more. We know that during sleep the lower centres are in activity, which is less restrained than during the waking state. We should not, indeed, conceive this to be the case with the equilibrial centre. No influence from the eyes can act upon it, or from any active state of the muscles of the legs. go on, and their effect on the centre will be in relative preponderance in consequence of the cessation of other impressions; they will also vary with every involuntary change in the position of the head, altering the direction of the pressure of the endolymph.

It once chanced to me to have an instructive illustration of the influence of altered pressure. All know, by occasional experience, the strange sleep sensation of falling - of going down, down some deep space. It often wakes the sleeper, and once woke me up so promptly that I caught the sound of what, I doubt not, was its cause. The sound was the flutter in one ear which is caused by contraction of a tympanic muscle, doubtless the stapedius. This lessens the pressure in the labyrinth, and the ampullae of the canals being at their lower ends, the pressure in them would be lessened by descent, and its diminution by the stapedius would suggest descent. It would seem, therefore, that even during sleep the equilibrial centre and also the cortex are readily in fluenced by impressions from the semicircular canals, although the auditory impulses, produced by sound, have little effect.

One example of attacks coming on during sleep has been already mentioned. Another was a lady of 33, in whom sudden vertigo had occurred for a year. There was a sense of motion in herself and objects, and if standing she. was sure to fall. During sleep she was awakened by a peculiar "grinding" sensation in the head, and a feeling that she was sinking through the bed. She dared not raise her head, and even when the room was dark she had the impression that objects about her were moving. Although she had a brother who was epileptic her vertigo was certainly aural. Deafness and hissing tinnitus had existed on the right side for eighteen months. Her objective vertigo was of the rather unusual form in which objects seem rotating around an axis in the line of sight, in this case in the direction opposite to the movement of the hands of a clock. It might conceivably be the result of isolated irritation of the right posterior semicircular canal, in which such actual rotation of the head would cause increased pressure.

\section{Extension of Symptons.}

Such symptoms as the sensations in the head we have considered, are of great importance, because they do not suggest the purely vertiginous nature of the attacks. The sudden "rush" to the head, or other sensations of which examples have been mentioned, are, as I have said, sug gestive of epilepsy. This is true also of cases in which there is an extension of such a sensation. Such extension may seem less strange if we remember how wide are the relations of the centre for equilibration. Its olosest connexion seems to be with the gastric centre of the vagus, 
shown by the nausea and vomiting that so often attend vertigo. The relation varies in different persons, as is sh iwn by their liability to sea-sickness, which is almost certainly produced through the agency of the semicircular canals. It is not surprising that the cardiac centre should also share the disturbance, with irregular and feeble action of the heart as the result. The vasomotor centres are likewise often disturbed; sudden nausea is of ${ }^{4} \mathrm{en}$ attended with perspiration, and the coldness of the surface, with or after vertigo, testifies to the vasomotor spasm.

The diffusion of the sudden sensory disturbance often gives rise to concern and perplexity. It was so in a man of 44, who had suffered for four years from tinnitus, referred to the right ear and head; never to the left ear. Usually hissing, it sometimes changed to a loud whistle for a few seconds. In spite of this evidence of irritation, his hearing was good. For a year he had been troubled with giddiness, and recently sudden exacerbations had occurred, in which he seemed to spin rapidly to the left, and sometimes fell; the onset of the sense of spinning was attended by a pain at the vertex, as if he had received a blow there. Note that it was not the sense of an impulse frum a blow, as in the cases I have before spoken of ; it was a sensation of the pain a blow would cause, and this passed, like a shcek, to the body, the arms, and the legs. It was felt at the heart as a sickening pain, which caused a feeling "as if he were going to die at once." After a second or two it ended as suddenly as it began, but the pain at the vertex lasted longer, and the feeling of rotation for a considerable time slowly lessening. It was ten minutes before he could stand, and for an hour the sensation of turning to the left was such that he could only walk with help. He was an intelligent man, and the account of his symptoms was clear. I saw him again after four years; the giddiness had ceased under the treatment, and then recurred in slighter form, still objective and subjective to the left, with a tendency to fall to that side. The tinnitus was unchanged. A year later the symptoms continued, but in slighter form. He was then suffering from gouty neuritis in"one arm.

\section{BORDER-LINE EPILEPSY.}

Before we consider the more definite relation to epilepsy that aural vertigo may present, it is important to note that true epileptic attacks may begin with the symptoms characteristic of labyrinthine giddiness, tinnitus, and vertigo. I have elsewhere described examples of this, ${ }^{1}$ but the following case is specially instructive, because there is strong reason to regard it as the result of old stationary damage to the brain, occurring during infancy. The patient was an intelligent girl of 18 , who had a severe brain illness in India at the age of 11 months. This began with a series of severe convulsions, and she is said to have been unconscious for a fortnight, and was then unable to move one arm and leg for a few days, after which their strength returned. It was thought that the convulsions were chiefly on one side, but on which side was not remembered. Her epileptic attacks began at the age of 13, and had continued with increasing frequency. Each began with a sudden sense of movement of objects and of herself to the right, and a confused sound in the right ear; with these there was a slight sense of nausea. The warning lasted long enough to allow her to sit down. Then some vague feeling made her close her lips and swallow two or three times. This she remembered, but was not conscious of any peculiar taste or smell. She then lost consciousness, and made a gurgling noise in her throat, which she did not afterwards remember; her limbs were the seat of slight tonic spasm, $s$ imetimes with a few jerks of the arms. The whole attack lasted about half a minute, and she was at once quite well.

It is probable that this patient had a small spot of damage to the brain near the auditory centre in the first left tempero-sphenoidal convolution. This centre is excited by a sound which reaches the right ear, and the normal effect is a movement of the head to the right. The instant spread of the discharge to the motor region would cause a sense of movement to the right, and also an impression that objects opposite her were moving to the right. Indeed, the discharge may have involved the left motor region at the same time as the auditory centre. Spreading at once to the motor region of the other lemisphere in the mysterious way the epileptic discharge extends, it caused the sliglit spasm on both sides, without the deviation of the head and eyes, which would have occurred had the motor discharge in the left hemisphere reached a bigher degree before it spread to the right. Thus the perception of vertigo in such a case, and in the aural form, may both be regarded as due to activity in the same region of the brain, the motor cortex, the difference being in the nature of the activity. In epilepsy it is a primary discharge; in ear disease it is secondary, a result of the disturbance of the centre for equilibration. It must be remembered that all sensations of vertigo, and connected with it, can only be consciously perceived through the cortex of the brain, whatever be their precise mechanism.

We should note, however, that in the case of epilepsy just described, the resemblance to aural vertigo was made more complete by the slight nausea which the giddiness seemed to cause. In vertigo we refer this to the direct influence of the equilibrial centre on that of the vagus, but all lower associations must be repeated in the region of the cortex through which consciousness is influenced, and we can thus understand that any vertigo that is felt may induce associated nausea. But we can also conceive that such epileptic vertigo may have a downward influence on the equilibrial centre and its relations.

\section{Association of Aural Vertigo and Epilepsy.}

The facts just mentioned may enable us better to consider the occasional coexistence of epilepsy and labyrinthine vertigo, and the question of an actual relation between the two. We must remember that the two diseases may simply coincide; both may occur in the same person. Neither is a rare disease, and they must by mere chance sometimes be met with together, without a direct relation between them. When the epileptic attacks are of the minor form, which vertigo may resemble, the distinctive diagnosis requires special care. There is sometimes evidence that the relation is more than mere coincidence. The attacks, although of different nature, sometimes seem to exert an influence one on the other.

An illustration of the association of vertigo and epilepsy was presented by a member of our own profession, gouty, 53 years of age, whose brother was epileptic. He had suffered for two years from slight nerve deafness and tinnitus. On the left side he could hear no note above 2,000 . For four months sudden attacks of giddiness had occurred; objects seemed moving to the left, and he fell to the left unless he could support himself, but he never lost consciousness. The intense giddiness lasted $1 \frac{1}{2}$ minutes, and for the same time objects seemed to oscillate laterally. He was never sick. For years, every three or four months, he had had a sudden sensation of a pleasant smell, like jasmine, which seemed to fill his nose and head, and lasted thirty seconds, without mental change. Sometimes it was followed by a brief but distressing sense of fullness in the throat and head, so intense that he felt as if "something must give way there." This feeling occurred independently three or four times a week, by night as well as by day, and at night it was associated with a sense of falling and a sound in the ear. (This seems similar to the stapedius effect I have already mentioned.) The sudden brief sense of smell must be regarded as a form of minor epilepsy, and there was some evidence that he once had a definite convulsive seizure.

A more direct relation between aural vertigo and epilepsy seemed to exist in a patient who probably inherited a tendency to the latter, since his father was epileptic. He was a man of 38 , and had become deaf to the watch on both sides, alike through air and bone. On the left, he could hear no tuning-fork; on the right, $\mathrm{C}^{3}(2,048)$ was audible only through the air; lower notes also through the bone. For five years there had been tinnitus, bilateral, but greater on the left side, and with it began attacks of giddiness, sudden in onset, lasting an hour or more, att-nded by vomiting. After two years they became very brief. There was a sudden sense of movement of objects to the left; he fell, and was unconscious for a moment, but the attack was over in two or three seconds. Similar attackss occurred in bed, with a feeling that he was turning to the right, and sometimes there was also momentary loss of consciousness. Subsequently, he became liable to attacks resembling minor epilepsy still more clcsely. They began with what he called 
a " numb sensation" passing up the spine to the head. If he had time to nip the skin at the back of the neck, this would arrest the attack, just as a warning sensation passing up the arm may be stopped by the same expedient. When the sensation reached the head it became "thrilling, like a galvanic battery," and often there was brief loss or consciousness. This case certainly presents the aspect of the development of minor epilepsy from what we may term "epileptoid vertigo," and this from the ordinary form.

A remarkable combination of aural vertigo and epilepsy was presented by another patient, whose symptoms were so remarkable as to deserve detailed description. She was a married woman of 35 , who had been slightly deaf all her life, and had suffered from tinnitus as long as she could remember. It became worse after an attack of scarlet fever at 18. At 20, she began to suffer from attacks of giddiness and sickness, each lasting five minutes. At 23 she had a fall on the ice, striking her head. At 32 she began to have attacks of minor epilepsy, which continued, occurring in the night as well as in the day, and at 33 severe nocturnal attacks began. But the tinnitus also went on, and so did brief attacks of giddiness. The minor attacks of epilepsy consisted of lip-smacking and loss of consciousness; at night the sound of the lips always aroused her husband. The severe attacks always began with the same lip-smacking, which was followed by deviation of the head to the right and by spasm-first tonic and then clonic-said to be confined to the right side; after each fit the right arm was weak for a time. There was never tongue-biting, but always micturition. There was no remembered sensation of smell or taste, but a remarkable effect of the severe attacks was complete loss of perception of flavours for two or three days. It was called "loss of taste"; but sweet and bitter were perceived, so that the loss must have been of the olfactory sensation which constitutes "flavour." Yet smell was not lost. I have pointed out " that tre warnings of epilepsy indicale a difference in the cerebral representation of olfactory impressions, according to their production through the anterior nares, as a smell or through the posterior nares, as a flavour. Although produced through the agency of the same nerve, they are always clearly distinguished by the epileptic patient who experiences one or the other.

Her attacks of giddiness seemed to have no relation to her epileptic seizures, except that there was a sudden sense of turning in the same direction, to the right, compelling her to hold on, and of movement of objects in the same direction. With this there was a sound of a shrill whistle going through the head from left to right, "like a train whistling while passing a station"; it gradually died away. These attacks were very brief, but they occasionally caused her to fall. A dazed feeling attended them when severe, and once or twice she lost consciousness. They were always followed by brief vomiting. In one she heard the whistle, and felt as if falling down a tremendous height; then she found herzelf on the floor, having hurt herself in the fall; she was still very giddy and vomiting The sickness ceased in about two minutes; the giddiness lasted five, and ended suddenly, as usual. Sometimes, as the tinnitus was passing away, it changed to a sound of bells ringing. A curious fact is that she sometimes had a peculiar taste in the mouth at the onset of these attacks of vertigo, although never with the epileptic seizure, in spite of the smacking of the lips. The attacks of giddiness had a close resemblance to the paroxysms of aural vertigo, and the fact that they began some years before the epileptic seizures, and continued distinct from the latter, gives some support to the opinion that this was their original nature. But their frequent repetition must have induced a central state, facilitating their recurrence, and it is possible that they had become purely central in nature. The features of the double series of attacks render the case one of unusual interest. The probability that the process had become largely central is important in relation to the next and last class we have to consider.

Pseudaural Vertigo.

One group of cases remains for consideration. They are very rare but of great clinical importance. Patients sometimes suffer from attacks similar in character to those caused by labyrinthine changes but present no permanent evidence of such disease. We have seen that in most cases there are tinnitus and impaired hearing to justify the diagnosis of ear-giddiness, but that in some the same opinion is warranted by the existence of tinnitus, since this, if persistent and pronounced, is evidence of labyrinthine irritation. In the cases now to be mentioned even this is absent as a permanent symptom. The symptoms consist only of the paroxysmal attacks, yet these bear the closest resemblance to those met with in the cases with unequivocal evidence of changes in the ear. For the present we may term them "pseudaural," leaving their exact nature an open question.

A man of 38 presented a pertinent example of this condition. His attacks were attended by a cephalic sensation similar to those we have considered. They occurred about once a fortnight. A sudden sensation passed over the head from behind, and was instantly followed by slight deafness, hissing tinnitus, and a sensation that objects before him were moving from left to right while he seemed still. It seemed to him to last about two minutes; nausea followed but no vomiting. Sometimes brief tinnitus occurred alone, and sounds for the moment seemed distant; there was no constant tinnitus and no impairment of hearing. It may be thought that such attacks might be caused by a contraction of a tympanic muscle, but, if so, there must have been considerable central co-operation.

It will be noted that, in the case just described, the symptoms were not only paroxysmal, but brief. So it was in a lady of 35 , with gouty ancestors. Her attacks occurred at intervals, varying from a day to four or five weeks, and were brought on by excitement, over-fatigue, and mental exertion. Her description was this: Quite suddenly she feels that the ground is rising up to her, and that she is going backwards. This seems to her to be due to some physical force pulling her back. With this there is a "rushing" sensation in the head. The feeling of going back lasts for two or three minutes, and its diminution is accompanied by a sound of rushing water in both ears, which ceases when she feels steady. Nausea follows, often for hours, but as a rule she does not vomit. Generally there is no impairment of consciousness, but twice it was lost in an attack-it is said for as long as five minutes. There was never micturition or any evidence of convulsion. A curious feature was that if she resisted the tendency to go back the sense of giddiness was more intense and afterwards, instead of mere nausea, she vomited for a couple of hours. At the early stage of the attack her face was pale and limbs cold; towards its close there was a little flushing. Her attacks were arrested by treatment, but recurred when it was omitted. Its resumption was followed by freedom for five years, when slighter attacks occurred of the same form, but without unconsciousness.

These paroxysmal symptoms were precisely such as we have seen are met with in other cases of certainly aural vertigo. Yet her hearing was good to every test, and there was no tinnitus apart from the attacks. (I have learned that she is still free from aural symptoms, although it is eleven years since I saw her first.) The sense of an external force dragging her back was analogous to the sense of pressure on the head, or of a blow, described in characteristic cases, and met with, as far as my observation goes, only in vertigo of labyrinthine origin. To ascribe the attacks to irritation absolutely limited to the semicircular canals, is an assumption scarcely justifiable, considering how constantly tinnitus of persistent character is associated with such irritation. Here a sound was only observed as part of the attack, and may have been due to extension of the central process. It is easier to conceive such attacks as purely central ; perhaps originally excited by labyrinthine irritation, but persisting independently. All peripheral associations are, as I have said, reproduced in the nerve centres, in the lower and also at the higher level of function, and we must not therefore attach to them undue weight as evidence of a distal cause. Such cases of pseudaural vertigo remain mysterious, even when all is said that can be, and they deserve careful study from those who chance to meet them.

The treatment of such cases as I have described is scarcely within the range of these lectures. Yet you may reasonably desire to know whether the facts and considerations I have put before you have any bearing on thera- 
peutics. They at least show more clfarly the reasons for the treatment that is customary. We have seen that the process of aural vertigo is a treble one, and that there are variations in the relative degree of its elements. One is the labyrinthine derangement, the second is the disturbance of the equilibrial centre, and the third is the process in the cerebral cortex thereby induced.

For the changes in the internal ear, as you are aware little can be done by direct treatment. The labyrinth is beyond the range of aural surgery. When its affection is secondary to middle-ear disease, the treatment of this is obviously the first consideration, but for the internal ear little can be done locally beyond the customary, and by no means useless, counter-irritation. It is always important to discern whether any diathetic state underlies the labyrinthine changes, and to treat it, in the hope of at least staying their further increase. A gouty condition, especially that which is inherited, is often to be traced. We know how frequently this induces changes in the fibrous tissues to which the membranous labyrinth belongs. Structural changes that have taken place we cannot hope to remove, and the tinnitus thus produced, although often lessened by treatment, seldom ceases, except in the rare cases in which the disease is so progressive as to abolish all aural function.

The study of the symptoms has brought prominently before us the part taken by the central structures in determining the occurrence and character of the attacks of vertigo, and it is on these that most influence can be exerted. We have seen that the suddenness of onset must be referred to the disturbance of the stability of the equilibrial centre, and bromides certainly enable us to lessen the readiness with which it is deranged, to reduce the frequency of the attacks, and also their severity. Several of the cases I have mentioned afford evidence of this effect, and in some, arrest continued as long as treatment was maintained. We may, at least provisionally, conceive the process as an upward "discharge," acting on the cerebral cortex, inducing in it motor and sensory activity, by which consciousness of the features of the attack arises. Although the assumed upward discharge of the equilibrial centre may differ in character from the sudden "discharge" of epilepsy, we can understand that it may be amenahle to the same influence. The effect of bromide seems sometimes to be increased by sedative agents, such as gelsemium and hyoscine.

In general, this treatment is effective in proportion to the degree in which the symptoms seem determined by the central instability, as indicated by the uniformity of the features of the attacks. It is least in the cases in which the aural symptoms present evidence of steady increase, and the attacks are variable in character.

My chief object has been to impress on you the features presented by these borderland cases of vertigo which resemble epilepsy, and to enable you, by the examples put before you, to discern the differences, and also the probable relation, between the symptoms of the two diseases. Yet a careful consideration of them, briefly as the cases are described, may subserve another object. Giddiness is a common symptom, usually passed as such without endeavour to learn its features or discover its cause. It would have been easy thus to dismiss the symptom in most of the cases I have described. They only yielded their instructive detail to careful investigation, and they should teach us to "call nothing common," since within that which seems to be so may lie much that is unfamiliar, much that will well reward our careful study.

1 Epilepsy, second edition, p. 234.2 Ibid., p. 73.

\section{A NOTE ON THE EFFECT OF BODILY EXERTION ON THE OPSONIC INDEX OF HEALTHY PERSONS.}

By G. G. ELLETT, B.A., M.B.

(From the Pathological Laboratory, University of Cambridge.)

In the British Medical Journal for November 25th, 1905 , p. 1396, Drs. Meakin and Wheeler published a paper on a series of observations on the variation of the opsonic index after exercise of patients suffering from pulmonary tuberculosis. All, with one exception, showed a lowered opsonic index after walking. It is, of course, possible that some, if not the whole, of the variation might occur in healthy persons. Taking this into consideration, and having the opportunity of examining the blood of several men training for a boat race, I determined to put the matter to a preliminary test. The subjects might be taken as good types of men in normal health, and the exercise of rowing is rightly called exertion.

As the index had previously been studied in exercised tuberculous patients, I used the tubercle bacillus as my indicator. Samples of the bloods were collected in the early morning, before any exercise had been taken, and again in the afternoon, when the men had returned from their day's outing. With a view to eliminating any possible effect from the intake of food, the samples were taken, as far as could be assured, before meals. This precaution I was led to adopt from the observation of a slight fall in the opsonic index of my control subject after a meal. Wright's method of preparation of emulsion and dilution of serum were followed as closely as possible ; and the same control subject was used almost throughout the experiments. I may add that the serum of my control subject, as also that of myself, which was used on eeveral occasions, has throughout a considerable number of experiments given very consistent results.

In one case the blood was examined eleven times before exerce and nine times after exercise, and the indices averaged :

$\begin{array}{lllll}\text { Before exercise } & \ldots & \ldots & \ldots & \text { O.I } \\ \text { After } & & \ldots & 132\end{array}$

$\begin{array}{lllll}\text { After exercise } & \ldots & \ldots & \ldots & 1.17\end{array}$

In another' case the blood was examined five times before and five times after exercise, with average of indices:

$\begin{array}{lllll}\text { Before exercise } & \ldots & \ldots & \ldots & \text { O.I. } \\ \text { After } & \ldots & \ldots & \ldots & 0.78\end{array}$

The "before exercise" index is, I think, probably rather lower than it should be, for the reason that on one or two occasions the morning samples of blood were not mixed immediately on arrival at the laboratory; whereas the serum of the control subject was taken in every case just prior to the experiment, and was consequently fresh, and the "after exercise" serum was never more than one hour old. If this fact were taken into consideration, we should get a larger difference between the indices before and after exercise than is recorded in the figures given above.

In two other cases the blood was examined, but not with sufficient regularity to give any reliable results. These experiments, though few in number, took a considerable time to carry out. And though, naturally, they do not settle the point raised, they afford evidence that severe exercise has some influence in lowering the opsonic index even in healthy persons, in whom, of course, there can be no question of auto-inoculation, as is claimed in the case of the tuberculous patients.

IN his report on the trade of Germany, Mr. Oppenheimer, the British Consul-General for that country, gives some interesting statistics on the relative meat consumption of the Briton and Teuton. One of the most remarkable incidents of last year's record was the existence of what was practically an approximation towards a meat famine. Formerly the importation of animals into Germany was considerable. Now the free traffic in pigs from Russia, etc., is entirely forbidden, the importation of foreign cattle has practically ceased, and the duty on fresl meat has this year been raised from 17 marks per centner to 27 marks. The idea has been to encourage home production, in the belief that German agriculture could eventually succeed in producing a sufficient supply of meat for the population. This expectation has proved erroneous. The estimate has assumed a consumption of $40 \mathrm{kilos}$ rer head, but it seems that it amounted to as much as 50.4 kilos. This is the first year for which accurate statistics have been available, and they have revealed some curious facts as to the great differences in kind between the British and German consumption. With us the basis of meat food consists of beef, which constitutes 45.1 per cent. of the whole consumption. With veal and mutton added the figure is raised to 69.7 per cent., leaving only 30.3 for the meat from pigs. This is almost exactly one-half of what is consumed in Germany, where 60.1 per cent. of the consumption is pork, ham, bacon, etc., and only 39.9 per cent. beef and mutton. These fundamental differences, it is stated, are not exclusivaly to be explained by national habits, but are also dependent on prices. The Briton pays just three-fourths of the price given by,the German for his meat, with the jesult that the latter is largely induced to use the meat of pigs. 